\title{
The Mechanism of Fear at Behavior, Brain Circuits and Molecular Level
}

\author{
Yuting Chen ${ }^{1, a}$ \\ ${ }^{1}$ College of Biological Sciences, China Agricultural University, 100193, Beijing, China
}

\begin{abstract}
The study of "fear" can be traced back to the differences between "behaviorism" and "psychoanalysis" on cognition, while recent research on the learning and memory of fear by using modern cell and molecular biology experimental techniques has made our understanding of "fear" gradually multilevel and in-depth. At present, our research on fear can be divided into three levels, namely behavior, brain circuits and molecular. At the level of behavior, Pavlovian conditioning experiments have become the core method for studying fear, and behavioral approaches have been used for fear-related mental illnesses, such as the treatment of Post-Traumatic Stress Disorder (PTSD). At the level of brain circuits, the brain circuits with the amygdala as the core have gradually become clear, and there is also a two-system or one-system debate on how to recognize the fear process. At the molecular level, the Hebbian synaptic plasticity mechanism and neuromodulatory-dependent mechanism are used to explain the process of fear memory acquisition. Based on the current three levels of research, revealing how feelings of fear may generate is likely to play a guiding role in the further understanding of "fear" and treatment of related diseases.
\end{abstract}

\section{Introduction}

Our understanding of fear has gone through a process from shallow to deep. When it comes to the word "fear", non-professionals usually regard it as an emotional response involving consciousness. Looking back on the professional understanding of "fear", two core psychological schools in the early 20th century "behaviorism" and "psychoanalysis" respectively gave their own explanations for "fear". In behaviorism, scientists represented by John Watson abandoned the illusory "consciousness" in psychology, and no longer studied "fear" as an emotion; however, based on Pavlov's classical conditioned reflex experiment, they believed that fear was also a constructive conditioned reflex $[1,2]$. B.f. Skinner, another behaviorist, adopted operant conditioned reflex experiments, suggesting that when the consequences of an action were associated with an aversive stimulus, "fear" caused the experimental target to have a tendency to avoid the action, which also explained "fear" from the perspective of behavior [3]. In psychoanalytic school, "fear" was still considered as the existence at mental level. Sigmund Freud's "drives" theory was the core of the interpretation of "fear". According to the psychoanalysts O. Hobart Mowrer and Neal Miller, "fear" was a driving force that can be acquired, and the Pavlov conditioned reflex experiment is the process of acquiring this driving force [4].

Neither behaviorism nor psychoanalysis is clear or proves what is going on in the brain. The brain is a black box, and threatening signals are input into the black box, which outputs fearful emotions or behaviors. With the development of modern cell and molecular biology technology, as in many cognitive processes, our research on "fear" is further deepened from the psychological level to the level of brain circuits involved in "fear", and even to the level of molecular mechanism regulating "fear" learning and memory. This thesis will illustrate the current understanding of learning and memory of fear from a multi-level perspective.

\section{Behavior Level}

With the end of the era of behaviorism, the performance of fear at the behavior level is no longer a hot issue in research. Therefore, when introducing the fear at behavioral level, the focus of this paper is not on the mechanism, but on two applications in which the behavior level research of fear is still active today: behavioral experiments and behavioral methods for the treatment of mental illness. The former is the basis of experimental research in the field of fear, and the latter is the practical significance of fear research.

\subsection{Conditioned reflex experiment of fear}

Behavioral experiments are still universally used to study learning and memory processes at all levels. Behavioral experiment is the process of initiating learning and memory macroscopically, and behavioral index is a method to test the effects of learning and memory. When studying the learning and memory of fear, what is the most commonly used is the conditioned

$\overline{{ }^{a} \text { Yuting Chen: chelseachen_yt@cau.edu.cn }}$ 
reflex experiment of fear. Rats are usually selected as experimental animals at present. The specific experimental methods are as follows. On the first day, let the animals adapt to the experimental environment. On the second day, the coupling of conditioned stimulus and non-conditioned stimulus is completed, in which the non-conditioned stimulus is electric shock, and the conditioned stimulus is sound. The coupled sound and electric shock stimulations usually last 1-5 times. The conditioned reflex of fear is established very quickly, and the effects of conditioned reflex can usually be tested on the third day. The method is that only giving conditioned stimulus - auditory-conditioned stimulus can induce fear related behavior, including defensive actions (such as stationary), autonomic response (such as changes in heart rate and blood pressure), endocrine reaction (such as the secretion of stress hormones), reduction of sensitivity to pain and reflex activities (such as startle reflex and winking reflex). At present, commonly used fear conditioned reflex experiments usually select animal stationary time length (freezing time) as indicators that show fear. On the third day, the duration of the freezing time is longer than that of relaxing, which indicates that the animal completes the learning of fear through the conditioned reflex experiment and obtains the memory of fear $[5,6]$.

\subsection{Solving fear-related human diseases based on behavior level - Post-Traumatic Stress Disorder (PTSD)}

It has been determined that many mental disorders of humans are closely related to the learning and memory process of fear, the most typical of which is PTSD. Traumatic events can induce strong physiological responses and emotions of fear, such as post-traumatic stress disorder, that is, reexperiencing, avoidance and hyperarousal of traumatic events exist for a long time and don't fade away, thus forming PTSD mental illness [7].

The current thinking of treating PTSD is based on several aspects of memory. For any learned memory, the brain first gets memory through acquisition, then conducts consolidation for memory, and then the memory may change during reconsolidation. Extinction is the implementation of memory removal at the behavioral level through the uncoupling of conditioned and non-conditioned stimulus. Cognitive behavioral therapy, the most common current treatment, is to treat PTSD from the idea of "extinction". However, this method does not actually eliminate fear memory and can only delay the start time of PTSD response [8]. By contrast, interfering with "consolidation" may be a more effective method. Three courses of long-term exposure interventions that begin about 12 hours after the trauma can effectively alleviate PTSD symptoms [7], but because a high proportion of patients do not seek medical treatment promptly after post-traumatic stimulation, making the use of a brief "consolidation" phase to treat PTSD not really feasible.

\section{Brain Circuits Level}

There are many research findings on the mechanism of fear at the level of the brain circuits. So far, it has been concluded that the conditioned reflex of fear has a complete brain circuit, with the amygdala as the core, and multiple brain areas get involved together. The deepening understanding of the brain circuits of fear has led to the emergence of two new theoretical models after psychoanalysis and behaviorism, both of which attempt to present the nature of fear, but there is no definite evidence to prove which is right and which is wrong.

\subsection{Brain circuits of fear conditioning}

Conditioned reflex experiments based on fear. Since the 1980s, many laboratories have been devoted to studying which brain regions fear conditioning activates and the connections between these regions, and have drawn relatively distinct brain circuits. Fear conditioning is established in the amygdala. Non-conditioned stimulus, such as electric shock, is introduced into the amygdala through periaqueductal gray (PAG), thalamus or cerebral cortex. Conditioned stimulus, such as sound signals, is first introduced to the area of the thalamus responsible for sound perception. It may be further introduced to the sound signal specific sensory areas of the cerebral cortex, which is then introduced into the amygdala. Lateral nucleus (LA) of the amygdala can achieve coupling of conditioned and unconditioned stimuli, especially the dorsal subnucleus (LAd), which is closely related to the synaptic plasticity in LA. This synaptic plasticity is governed by the projection between the basal amygdala (basal, B) and the limbic cortex (PL), B and ventral hippocampus (vHC). Then, LA projects the signals to the central nucleus (CE) of the amygdala, which can pass through the basal of the amygdala (basal, B), accessory basal (AB), and intercalated cell masses (ICM), and they can also reach the central nucleus directly. CE can be further projected to the hypothalamus and brainstem to produce a fear response. CE projection to PAG may cause static immobility; projection to the lateral hypothalamus ( $\mathrm{LH})$ can initiate autonomic response via autonomic nerve descending conduction; projection to the paraventricular nucleus of the hypothalamus (PVN) can trigger the release of stress hormones $[5,6,10]$. The brain circuits involved in the "extinction" process of fear are mediated by different pathways of the same structure, where the input signals from the ICM to the infralimbic cortex (IL) and to the BA and ICM lead to the decrease of output signals from $\mathrm{CE}$ to hypothalamus and PAG, making the fear response weakening [9].

Depending on whether or not it passes through the cerebral cortex, the pathways where input signals reach the amygdala can be divided into two types, namely high road and low road. The low road refers to the direct transmission of sensory signals to the amygdala after reaching the sensory processing area of thalamus. The high road refers to that the sensory signals reach the hypothalamus, continue up to the sensory areas of the cerebral cortex, and then project from the cerebral cortex 
to the amygdala. The existence of low road means that threatening signals can directly trigger a series of fear responses without going through the cerebral cortex. There is no consciousness participation in this process, and the processing of information is rough, rapid and transient. The high road, which involves the delicate processing of sensory by the cerebral cortex, is relatively slow but lasts a relative long time [11].

Previous studies have established the core position of the amygdala in fear learning and memory. In recent years, research has paid more and more attention to the functions of various sub nucleus in the amygdala and other brain areas that may be involved in fear learning and memory, and the research subjects have gradually shifted from model animals to humans [7]. For example, some studies have used functional magnetic resonance imaging (fMRI) to examine the role of sub nucleus of the amygdala in fear and anxiety, and found that threatening signals could cause the excitation of bed nucleus of stria terminalis (BST) [12-14].

\subsection{Re-understanding fear based on the level of brain circuits}

The above study of fear-related brain circuits reveals that fear can be conscious or unconscious. On this basis, scientists represented by Joseph LeDoux put forward the two-system framework of fear. The core of this model is that fear at cognitive, physiological and behavioral level is caused by two different generators, or systems controlled by different brain circuits that are relatively fragmented, and it is suggested to use the concept of "fear" only at cognitive level. Instead, it should be replaced by defensive response at physiological and behavioral levels.

The two-system model emphasizes that fear is conscious, which makes some scientists think that the model is related with psychoanalysis. As a result, they rather support the central fear generator framework, also known as the one-system framework. The core of this model is that the fear at cognitive, physiological and behavioral level is different effect triggered by the same central generator, controlled by a common central brain circuit. They also consider that the concept of "fear" should be a comprehensive reaction including emotion and physiology [15]. Both models are currently lack of evidence.

\section{Molecular Level}

Hebbian synaptic plasticity mechanism (Hebbian Mechanisms) and neuromodulatory-dependent mechanisms are two core principles of conditioned reflex of fear. Recent studies using optogenetics have revealed that Hebbian Mechanisms is essential, but not sufficient, for the formation of fear associative memory, and must be cooperated with a neuromodulatory mechanism [16]. This section only introduces the relevant mechanism of fear conditioning establishment, which is equivalent to the process of acquisition of memory. As for consolidation, reconsolidation, and extinction, the molecular mechanisms of these processes have also been studied, but this paper does not elaborate on them.

\subsection{Hebbian synaptic plasticity mechanism (Hebbian Mechanisms)}

The core of Hebbian model is the signal transmission at the synapse and signal transduction in postsynaptic neurons. The same is true for the formation of fear memories. The n-methyl-d-aspartic acid receptor (NMDARs) located on the postsynaptic membrane of LA is a ligand-gated $\mathrm{Ca} 2+$ channel, and the recognized ligand is the glutamate released from the presynaptic membrane [6,17]. It is found that the injection of antagonist ifenprodil into LA of amygdala can block the subunit NR2B of NMDARs and inactivate NMDARs in LA, resulting in the interference of fear learning process [18]. In addition, experiments have suggested that the silence of neuroligin-1 expression in amygdala mediated by virus may eventually lead to the formation of associative memory that fails to realize fear by reducing the number of NMDARs. NMDARs bind to ligand and cause the opening of postsynaptic membrane channels, which leads to the internal flow of $\mathrm{Ca} 2+$ and the increase of intracellular calcium ion concentration, thus giving the $\mathrm{Ca} 2+$ dependent calmodulin kinase II (CaMKII) activity due to autophosphorylation. In LA, autophosphylation of CaMKII may significantly increase the number of dendritic spines, which means that more synapses are built, while blocking the activity of CaMKII can lead to the failure of forming fear memories [20]. Active CaMKII can activate numerous events in the cell, among which serine 831 of phosphorylation $\alpha-$ amino-3-hydroxy-5-methyl-4-isoxazole-propionic acidtype glutamate receptor (AMPAR) is directly related to overhang formation. AMPAR receptor gets phosphorylation and migrates to near the cell membrane, which can directly cause the increase in synaptic strength. The experiment of knocking out AMPAR receptor subunit in LA also indicates its necessity in the process of memory formation of fear.

In addition, GABAergic interneuron in LA plays a significant role in regulating synaptic plasticity. Previous studies have found that activation of $\mathrm{GABA}_{\mathrm{A}}$ receptors in LA might lead to the blocked acquisition of fear memories [21, 22]. Later studies have found that blocking the $\alpha 1$ subunit of GABA receptor interfered with the formation of fear memory. This was due to the presence of GABA heterodimers on cells that did not express the $\alpha 1$ subunit. Blocking the $\alpha 1$ subunit, however, caused these GABA heterodimers that did not express the $\alpha 1$ subunit to accept more ligand. On the contrary, the signal transmission efficiency was enhanced [23].

\subsection{Neuromodulatory-dependent mechanism}

Neuromodulatory-dependent mechanism can be divided into two kinds, one of which is Monoamine neurotransmitter dependence, and another mechanism is 
Metabolic glutamate receptor mediation. Among them, the Monoamine neurotransmitter dependence mechanism involves Norepinephrine (NE) and dopamine (DA). The interaction between Norepinephrine (NE) and $\beta$ adrenergic receptor $(\beta-\mathrm{AR})$ is more influential in this mechanism. It is reported that block of the $\beta$-adrenergic receptor $(\beta-A R)$ in LA can causally lead to blocked formation of fear memory [24]. On the contrary, the activation of $\beta$-adrenergic receptor ( $\beta$-AR) in LA can promote the formation of fear memory by the regulation of Hebbian mechanism [25]. The role of the $\alpha 1$ adrenergic receptor, which acts as a receptor for NE, is just the opposite of $\beta$-AR. The blockade of this receptor promotes the formation of fear memory [26]. DA also promotes the formation of fear memory. Activation of DA receptors in the amygdala (including D1 subtypes and D2 subtypes) can induce an increase in the formation of fear memory [5].

Metabolic glutamate receptors (mGluRs) can regulate synaptic plasticity, which further affects the process of learning and memory formation of fear, but is not directly involved in the Hebbian mechanism. The activation of class I mGluRs and class III mGluRs in LA has been found to be necessary in the formation of fear memory [27-30].

\section{Conclusion}

A large part of current research on learning and memory of fear lacks in the interpretation of "sense of fear", namely fear at the cognitive level, or our emotions of fear. Only psychoanalysis offers systematic and detailed explanation of fear. This is because psychoanalysis attaches great importance to the concept of "consciousness". Therefore, when they explain fear, they tend to think that "fear" is "sense of fear" itself, and rationalize "fear" with driving force theory. However, the basic method of psychoanalysis research relies on subjects' subjective description of their emotions, which makes the theory of psychoanalysis not widely accepted in the academic world.

In fact, our current understanding of fear at the behavioral, brain circuits, molecular level is based on the conditioned reflex experiments of fear. Fear conditioning experiments can directly reflect the brain's learning and memory process of fear. Since the end of the twentieth century, the study has shown that at the level of brain circuits, the amygdala in LA is the critical area of fear learning and memory. The conditioned or nonconditioned threat signals transmit from the thalamus, cerebral cortex to LA. After the coupling is achieved (mainly) in LA, they reach CE via or without some intermediate cerebral nuclei, and further project into the hypothalamus, periaqueductal gray, etc., resulting in a series of fear responses at the behavioral level. The molecular mechanism is mainly used to explain how the LA region achieves the coupling of conditioned and nonconditioned stimuli. The Hebbian synaptic plasticity mechanism and the neuromodulatory mechanism jointly realize the formation process of the associative fear memory, that is, the learning process of fear.
The conditioned reflex experiment of fear is with obvious tendency of behaviorism. Using experimental animals represented by rats, we can only pay attention to the behavioral results of fear, but we cannot say whether the rat has a "sense of fear" and to what extent. This makes a series of results from the conditioned reflex experiments bring many problems when applied to humans. Clinical studies of PTSD have found that "extinction" experiments, which have long been thought of as able to remove fear memory (conditioned and nonconditioned stimulus uncoupling), do not fundamentally achieve the aims of eliminating memories and reducing the sense of fear in the treatment of patients with PTSD. Other studies have found that a large number of the drugs currently used to treat anxiety are actually designed to control human's behavioral response to fear, rather than reduce the "sense of fear" [11]. The first reason for this problem is that the conditioned response experiment is defective in interpreting the fear at cognitive level. The use of experimental animals means that we cannot evaluate their feelings, but only observe their behaviors. Secondly, both conscious and unconscious signals processing are carried out simultaneously in the brain, which can cause physiological and behavioral changes among people who are in fear. It remains to be seen whether behavioral techniques such as "extinction" can alter unconscious fear brain circuits. The third reason is that it is not clear what the emotional nature of fear is. Some people are convinced that "emotion" is a comprehensive effect. It is a combination of a series of behaviors and signal transmission in the circuits, which triggers the brain to judge its own state. In order to truly understand "fear" and realize the treatment of related mental diseases, in addition to the further study of the learning and memory of fear at behavior, brain circuits and molecular level, it may be of great significance to interpret "sense of fear" beyond psychology.

\section{References}

1. Watson, J. B. \& Rayner, R. Conditioned emotional reactions. 1920. The American psychologist 55, 313317 (2000).

2. Watson, J. B. Behaviorism. (W.W. Norton, 1925).

3. Skinner, B. F. The Behavior of Organisms: An Experimental Analysis (AppletonCentury-Crofts, 1938).

4. Mowrer, O. H. \& Lamoreaux, R. R. Fear as an intervening variable in avoidance conditioning. Journal of comparative psychology 39, 29-50 (1946).

5. Johansen, J. P., Cain, C. K., Ostroff, L. E. \& LeDoux, J. E. Molecular mechanisms of fear learning and memory. Cell 147, 509-524, (2011).

6. LeDoux, J. E. Emotion circuits in the brain. Annual review of neuroscience 23, 155-184, (2000).

7. Kearns, M. C., Ressler, K. J., Zatzick, D. \& Rothbaum, B. O. Early interventions for PTSD: a review. Depression and anxiety 29, 833-842, (2012). 
8. Sijbrandij, M. et al. Treatment of acute posttraumatic stress disorder with brief cognitive behavioral therapy: a randomized controlled trial. The American journal of psychiatry 164, 82-90, (2007).

9. Maddox, S. A., Hartmann, J., Ross, R. A. \& Ressler, K. J. Deconstructing the Gestalt: Mechanisms of Fear, Threat, and Trauma Memory Encoding. Neuron 102, 60-74, (2019).

10. Fanselow, M. S. \& Poulos, A. M. The neuroscience of mammalian associative learning. Annual review of psychology 56, 207-234, (2005).

11. LeDoux, J. Anxious: Using the Brain to Understand and Treat Fear and Anxiety. (Viking, 2015).

12. Fox, A. S., Oler, J. A., Tromp do, P. M., Fudge, J. L. \& Kalin, N. H. Extending the amygdala in theories of threat processing. Trends in neurosciences 38, 319-329, (2015).

13. Somerville, L. H., Whalen, P. J. \& Kelley, W. M. Human bed nucleus of the stria terminalis indexes hypervigilant threat monitoring. Biological psychiatry 68, 416-424, (2010).

14. Mobbs, D. et al. Neural activity associated with monitoring the oscillating threat value of a tarantula. Proceedings of the National Academy of Sciences of the United States of America 107, 20582-20586, (2010).

15. Fanselow, M. S. \& Pennington, Z. T. A return to the psychiatric dark ages with a two-system framework for fear. Behaviour research and therapy 100, 24-29, (2018).

16. Johansen, J. P. et al. Hebbian and neuromodulatory mechanisms interact to trigger associative memory formation. Proceedings of the National Academy of Sciences of the United States of America 111, E5584-5592, (2014).

17. Malenka, R. C. \& Nicoll, R. A. Long-term potentiation--a decade of progress? Science $\mathbf{2 8 5}$, 1870-1874 (1999).

18. Rodrigues, S. M., Schafe, G. E. \& LeDoux, J. E. Intra-amygdala blockade of the NR2B subunit of the NMDA receptor disrupts the acquisition but not the expression of fear conditioning. The Journal of neuroscience : the official journal of the Society for Neuroscience 21, 6889-6896 (2001).

19. Kim, J. et al. Neuroligin-1 is required for normal expression of LTP and associative fear memory in the amygdala of adult animals. Proceedings of the National Academy of Sciences of the United States of America 105, 9087-9092, (2008).

20. Rodrigues, S. M., Schafe, G. E. \& LeDoux, J. E. Molecular mechanisms underlying emotional learning and memory in the lateral amygdala. Neuron 44, 75-91, (2004).

21. Muller, J., Corodimas, K. P., Fridel, Z. \& LeDoux, J. E. Functional inactivation of the lateral and basal nuclei of the amygdala by muscimol infusion prevents fear conditioning to an explicit conditioned stimulus and to contextual stimuli. Behavioral neuroscience 111, 683-691 (1997).

22. Wilensky, A. E., Schafe, G. E. \& LeDoux, J. E. Functional inactivation of the amygdala before but not after auditory fear conditioning prevents memory formation. The Journal of neuroscience : the official journal of the Society for Neuroscience 19, Rc48 (1999).

23. Wiltgen, B. J. et al. The alpha1 subunit of the GABA(A) receptor modulates fear learning and plasticity in the lateral amygdala. Frontiers in behavioral neuroscience 3, 37, (2009).

24. McGaugh, J. L. Memory--a century of consolidation. Science 287, 248-251 (2000).

25. Johansen, J. P. et al. Optical activation of lateral amygdala pyramidal cells instructs associative fear learning. Proceedings of the National Academy of Sciences of the United States of America 107, 12692-12697, (2010).

26. Lazzaro, S. C., Hou, M., Cunha, C., LeDoux, J. E. \& Cain, C. K. Antagonism of lateral amygdala alpha1adrenergic receptors facilitates fear conditioning and long-term potentiation. Learning \& memory (Cold Spring Harbor, N.Y.) 17, 489-493, (2010).

27. Rodrigues, S. M., Bauer, E. P., Farb, C. R., Schafe, G. E. \& LeDoux, J. E. The group I metabotropic glutamate receptor mGluR5 is required for fear memory formation and long-term potentiation in the lateral amygdala. The Journal of neuroscience : the official journal of the Society for Neuroscience 22, 5219-5229 (2002).

28. Rudy, J. W. \& Matus-Amat, P. DHPG activation of group $1 \mathrm{mGluRs}$ in BLA enhances fear conditioning. Learning \& memory (Cold Spring Harbor, N.Y.) 16, 421-425, (2009).

29. Siegl, S., Flor, P. J. \& Fendt, M. Amygdaloid metabotropic glutamate receptor subtype 7 is involved in the acquisition of conditioned fear. Neuroreport 19, 1147-1150, (2008).

30. Schmid, S. \& Fendt, M. Effects of the mGluR8 agonist (S)-3,4-DCPG in the lateral amygdala on acquisition/expression of fear-potentiated startle, synaptic transmission, and plasticity. Neuropharmacology 50, 154-164, (2006). 